\title{
Acute Myocardial Infarction Associated with Pregnancy Successfully Treated with Percutaneous Coronary Intervention
}

\author{
Tadaaki Arimura, Ryoko Mitsutake, Shin-ichiro Miura, Hiroaki Nishikawa, Akira Kawamura \\ and Keijiro Saku
}

\begin{abstract}
We describe the case of a 43-year-old primiparous woman who had acute myocardial infarction. She underwent successful primary percutaneous coronary intervention. Elective cesarean section was performed uneventfully at 32 weeks gestation.
\end{abstract}

Key words: acute myocardial infarction, percutaneous coronary intervention, primiparous woman

(Inter Med 48: 1383-1386, 2009)

(DOI: 10.2169/internalmedicine.48.2208)

\section{Introduction}

Acute myocardial infarction (AMI) during pregnancy is a rare but serious condition, based on the danger of maternal mortality and fetal loss. Pregnancy has been shown to increase the risk of AMI about 3- to 4-fold (1). The incidence of AMI in pregnancy is expected to increase, due to the trend for childbearing at older ages and advances in reproductive technology, which enable many older women to conceive (2).

\section{Case Report}

A 43-year-old primiparous woman who presented with sudden-onset chest pain at 21 weeks gestation was admitted to our University Hospital 45 minutes after the onset of symptoms. She had dyslipidemia, but no history of other coronary risk factors, such as diabetes mellitus, hypertension, or smoking. In the lipid profile, the levels of triglyceride, HDL-C and LDL-C were $210 \mathrm{mg} / \mathrm{dL}, 50 \mathrm{mg} / \mathrm{dL}$ and $143 \mathrm{mg} / \mathrm{dL}$. There was no previous episode of connective tissue disease, vasculitis, an impaired anticoagulant mechanism (protein $\mathrm{C}$ deficiency, protein $\mathrm{S}$ deficiency), or antiphospholipid antibody syndrome, which are associated with a thrombotic tendency. Birth control pills had never been used. The electrocardiogram on admission showed ST elevation in leads V1-V6 (Fig. 1), and the echocardiogram showed that wall motion in the anterior region was severely reduced, consistent with AMI.

We consulted an obstetrician regarding the safety of administering clopidogrel and the use of radiation, and both were consequently considered to be safe because organogenesis was complete. Thus, we planned to use a bare-metal stent and clopidogrel for 2 weeks after percutaneous coronary intervention (PCI). While the symptom-to-door time was only 45 minutes, the door-to-needle time was $115 \mathrm{~min}$ utes because of this consultation.

Urgent coronary angiography showed total occlusion of the left anterior descending (LAD) coronary artery, thrombolysis in myocardial infarction (TIMI) grade 1 flow, without coronary dissection (Fig. 2). PCI was then performed. Thrombectomy using an aspiration device (Thrombuster III, Kaneka Corp., Osaka, Japan) was attempted and a red and white thrombus was retrieved. A $3.0 \times 20 \mathrm{~mm}$ bare-metal stent (Liberte, Boston Scientific Japan, Tokyo, Japan) was implanted to the LAD, and resulted in successful angioplasty and restoration of TIMI grade 3 flow. Needle-toballoon time was 30 minutes (total door-to-balloon time of 145 minutes). The combination of aspirin (100 mg/day) with clopidogrel (75 mg/day for 2 weeks) was initiated. Echocardiogram after PCI showed local asynergy of the anterior 


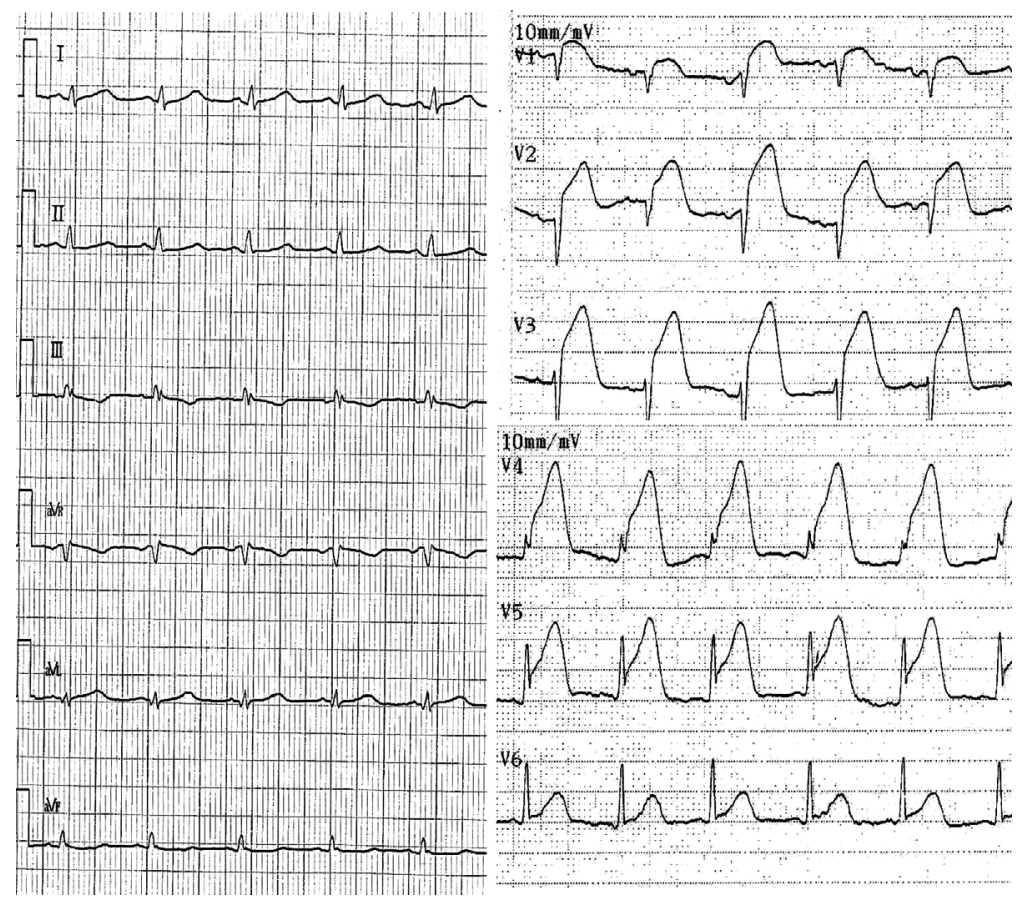

Figure 1. The electrocardiogram showed ST elevation in leads V1-V6.
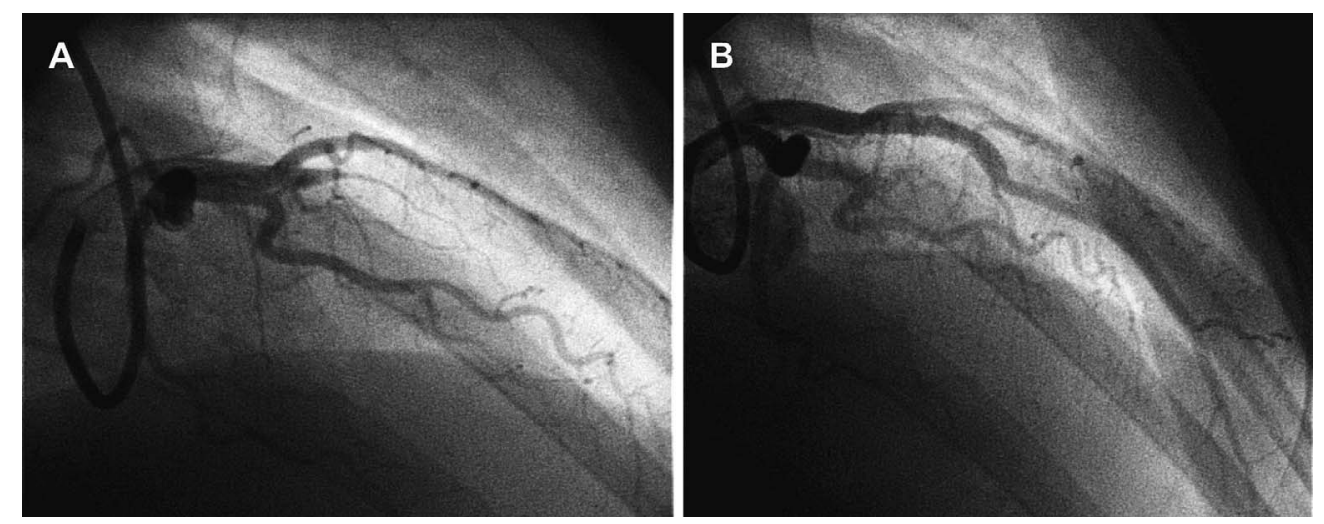

Figure 2. Left coronary angiography (RAO cranial). A) Total occlusion with thrombus of the proximal LAD. B) Successful PCI using aspiration thrombectomy and a bare-metal stent. RAO: right anterior oblique, LAD: left anterior descending coronary artery, PCI: percutaneous coronary intervention

wall with reduced left ventricular (LV) systolic function [LV ejection fraction $(\mathrm{EF}) 49 \%, \mathrm{LV}$ dimension in end-diastole (LVDd) $50 \mathrm{~mm}$. The serum levels of creatinine kinase peaked at 4,541 IU/L 6 hours after PCI.

Elective cesarean section was planned at 32 weeks gestation. Aspirin was stopped for a week before cesarean section, and heparin was initiated to prevent thrombotic complications after stent implantation. Echocardiogram before delivery showed reduced LV systolic function (LVEF 43\%, LVDd $52 \mathrm{~mm}$ ), but no evidence of congestive heart failure. Elective cesarean section was uneventful (blood loss 600 $\mathrm{mL}$ ), and a 1,967 $\mathrm{g}$ infant with an Apgar score of 9 was delivered. Heparin was replaced by aspirin postpartum. Both the mother and infant were discharged at 8 days postpartum.

Discussion

Pregnancy-associated AMI, which has been reported to occur in 3-10 cases per 100,000 deliveries, is rare $(1,3,4)$. A previous review demonstrated that pregnancy-associated AMI occurred at all stages of pregnancy and more commonly late in pregnancy, with a peak incidence during the peripartum or postpartum period (5). In the present case, the event occurred relatively early in the second trimester of pregnancy. The incidence of mortality in women who have been diagnosed with AMI during the peripartum period is twice as high as those who have been diagnosed in the antepartum and postpartum periods $(3,5)$.

A previous review revealed a relatively high incidence of known risk factors for AMI in patients with pregnancy- 
associated AMI, despite their young ages. Most of these patients $(72 \%)$ were older than 30 years (range 19 to 44 years). Ladner et al (3) identified hypertension, diabetes, advanced maternal age, preeclampsia, and eclampsia as independent risk factors for pregnancy-associated AMI, and James et al (4) also found that thrombophilia, transfusion, and postpartum infections were significant risk predictors for AMI. In the present case, the only risk factor for pregnancyassociated AMI was advanced maternal age (43 years), in addition to dyslipidemia as an established coronary risk factor.

Pregnancy-associated AMI has been thought to be related to both an excess procoagulant state and increased stress on the cardiovascular system, leading to coronary thrombosis or spontaneous coronary dissection. Since the anterior wall of the left ventricle is the most common location of pregnancyassociated AMI (5), reperfusion is a priority. The culprit lesion in this case was also in the LAD, and was caused by thrombotic occlusion without coronary artery dissection. Distal embolization has been considered to be a major cause of insufficient reperfusion. In this case, we used aspiration thrombectomy to prevent distal embolization, and successful angioplasty without no-reflow was achieved.

Recent recommendations for drug therapy in non-pregnant patients with AMI include the use of heparin, aspirin and clopidogrel (6). Heparin does not cross the placenta, and several reports have demonstrated that heparin is safe to use during pregnancy (7). A meta-analysis (8) has suggested that low-dose aspirin ( $<150 \mathrm{mg} /$ day) is safe during both the second and third trimesters. Information on the use of clopidogrel during pregnancy is very limited. In 6 previous case reports in which clopidogrel was administered during pregnancy (9-14), no complications regarding any adverse effects of clopidogrel on the fetus were observed. These drugs were also administered in our case without an adverse effect throughout pregnancy.

An elective cesarean section avoids a long or stressful labor and allows better control of the time of delivery as well as the presence of an appropriate medical team including an experienced obstetrician, obstetric anesthesiologist, cardiologist, and pediatrician. On the other hand, vaginal delivery eliminates the potential risks associated with anesthesia and a major surgical procedure, which include hemodynamic fluctuations, larger blood loss, pain, infection, respiratory complications, damage to pelvic organs, and potential unfavorable effects on future reproductive health. Ultimately, we considered that cesarean section was a better alternative.

The use of radiation during pregnancy should be kept to a minimum. Radiation-induced malformations during pregnancy have a threshold of 100-200 mGy or higher. Cardiac catheterization and PCI may also result in fetal exposure of $<10 \mathrm{mGy}$ regardless of the approach site. The pelvic region during cardiac catheterization received only $<1 \mathrm{mGy}$ in most cases as evaluated by thermoluminescent dosimeters (TLDs) (15). Thus, it may be possible to perform PCI for AMI during pregnancy as usual without any adverse effect on the fetus. Furthermore, since organogenesis in this patient was complete, PCI for this patient could be performed without shielding the abdomen against radiation.

In conclusion, the present patient underwent successful PCI for pregnancy-associated AMI, and delivered a healthy baby without complications. The incidence of pregnancyassociated AMI is expected to increase, due to developments in reproductive technology that are enabling many older women to conceive. Further studies on the management of pregnancy-associated AMI are needed.

\section{References}

1. Roth A, Elkayam U. Acute myocardial infarction associated with pregnancy. Ann Intern Med 125: 751-762, 1996 Review.

2. Paulson RJ, Boostanfar R, Saadat $P$, et al. Pregnancy in the sixth decade of life: obstetric outcomes in women of advanced reproductive age. JAMA 288: 2320-2323, 2002.

3. Ladner HE, Danielsen B, Gilbert WM. Acute myocardial infarction in pregnancy and the puerperium: a population-based study. Obstet Gynecol 105: 480-484, 2005.

4. James AH, Jamison MG, Biswas MS, Brancazio LR, Swamy GK, Myers ER. Acute myocardial infarction in pregnancy: a United States population-based study. Circulation 113: 1564-1571, 2006.

5. Roth A, Elkayam U. Acute myocardial infarction associated with pregnancy. J Am Coll Cardiol 52: 171-180, 2008 Review.

6. Antman EM, Anbe DT, Armstrong PW, et al. American College of Cardiology; American Heart Association; Canadian Cardiovascular Society. ACC/AHA guidelines for the management of patients with ST-elevation myocardial infarction--executive summary. J Am Coll Cardiol 44: 671-719, 2004.

7. Bates SM, Greer IA, Hirsh J, Ginsberg JS. Use of antithrombotic agents during pregnancy: the Seventh ACCP Conference on Antithrombotic and Thrombolytic Therapy. Chest 126: 627S-644S, 2004 Review.

8. Imperiale TF, Petrulis AS. A meta-analysis of low-dose aspirin for the prevention of pregnancy-induced hypertensive disease. JAMA 266: 260-264, 1991.

9. Sullebarger JT, Fontanet HL, Matar FA, Singh SS. Percutaneous coronary intervention for myocardial infarction during pregnancy: a new trend? J Invasive Cardiol 15: 725-728, 2003.

10. Shah P, Dzavik V, Cusimano RJ, Sermer M, Okun N, Ross J. Spontaneous dissection of the left main coronary artery. Can $\mathbf{J}$ Cardiol 20: 815-818, 2004.

11. Martín M, Romero E, Morís C. Acute myocardial infarction during pregnancy. Treatment with clopidogrel. Med Clin (Barc) 121: 278-279, 2003 (in Spanish).

12. Klinzing P, Markert UR, Liesaus K, Peiker G. Case report: successful pregnancy and delivery after myocardial infarction and essential thrombocythemia treated with clopidogrel. Clin Exp Obstet Gynecol 28: 215-216, 2001.

13. Nallamothu BK, Saint M, Saint S, Mukherjee D. Clinical problem-solving. Double jeopardy. N Engl J Med 353: 75-80, 2005.

14. Miller RK, Mace K, Polliotti B, DeRita R, Hall W, Treacy G. Marginal transfer of ReoPro (Abciximab) compared with immunoglobulin G (F105), inulin and water in the perfused human placenta in vitro. Placenta 24: 727-738, 2003.

15. Saito I. The present condition and the countermeasure against pa- 
Inter Med 48: 1383-1386, 2009 DOI: 10.2169/internalmedicine.48.2208

tient exposure in cardiac catheterization. Nippon Hoshasen Gijutsu

Gakkai Zasshi 58: 1016-1022, 2002 (in Japanese).

(C) 2009 The Japanese Society of Internal Medicine http://www.naika.or.jp/imindex.html 